INTERNATIONAL JOURNAL OF RESEARCHES IN BIOSCIENCES, AGRICULTURE \& TECHNOLOGY C VISHWASHANTI MULTIPURPOSE SOCIETY (Global Peace Multipurpose Society) R. No. MH-659/13(N) www.vmsindia.org

\title{
ULTRASTUCTURE OF EGGSHELL OF LIZARD Hemiductylus flaviviridis
}

Deshmukh V.A.

Yeshwant Mahavidyalaya, Wardha (M.S) India

\begin{abstract}
Majority of reptiles are oviparous. The shells structures differences are found according to their breeding ground in Hemiductylus flaviviridis eggshell is calcareous. The crystalline layer is well developed and forms a column. The outer structures shows presence of fibres on outer surface, in between these fibres crystals are seen. The shell membrane is non fibrilar porous and appear like that of sheet of organic material.
\end{abstract}

Keywords: Oviparous, Eggshell, Hemiductylus flaviviridis

\section{Introduction:}

Majority of the reptiles are oviparous but some retain the eggs internally and bear their young ones alive (viviparous). In the oviparous reptiles, the eggshell is well developed. The reptiles lay their eggs at different places as per required environmental conditions necessary for their incubation. During incubation, the eggshell plays an important role in exchange of gases, moisture and in excretion of metabolic waste.

The suborder Lacertilia and Ophidia, include lizard and snakes, possess both viviparous and oviparous populations. Eggshells of oviparous lepidosauria are either flexible, 'Parchment-like' or rigid, calcareous. And have both crystalline and fibrous components (Packard et al., 1982 a,b; Packard and Hirsch 1986; Blackburn, 1993, 1995; Mathies and Andrews, 2000). No other membrane seems to be present on the inner side of the shell of lepidosaurian eggs. Pores seem not to occur in eggshell of lepidosaurian (Packard, et al., 1977). The calcareous eggs are produced by many gekkonid lizards such as Hemiductylus turcicus and Lacerta agilis calcified (Jacobi, 1936).

The structure of eggshell is of prime importance for the developing embryo. Although India is rich in reptilian fauna representing the members of all existing sub-classes very little is known about their eggshells except few reports on turtle eggshell (Sahoo, et al., 1996). Therefore in the present investigation an attempt has been made to study the eggshell morphology of Hemiductylus flaviviridis using scanning electron microscope. Our observation would serve as a base line for investigating the effects of shell structure and strength on hatchling trauma, influence of maternal diet, gases exchange metabolic exchange and the evolution of viviparity.

\section{Materials and Methods:}

\section{Material:}

In the present study of eggshells the common wall lizards have been selected. Their eggs were collected from the nesting habitats during their respective reproductive cycle. The females of wall lizards, Hemiductylus flaviviridis lay clutches of 4 to 7 eggs on the perfectly dried substrate in dark places in the month of April to June. The eggs were collected from the loft of house, which is dark and a dry place.

\section{Preparation of Sample :}

The collected eggs were brought to the laboratory, at Department of Zoology. The samples were prepared scanning electron microscopy in the following manner.

The inner content of eggs were removed by breaking eggs and the eggshells were washed thoroughly with distilled water to remove any foreign material on the outer surface as well as yolk present on the inner surface of the eggshells. The eggshells were dried for over night in an oven at $35^{\circ} \mathrm{C}$ to remove the moisture.

After complete drying of eggshells the pieces of eggshells were processed for scanning electron microscopy. Pieces were affixed with adhesive on the metal stub used for scanning electron microscope (SEM) in the following manner. 
1) The outer surface: The fragments of eggshells were mounted on stub having outer surface facing up words upper most.

2) The radial or cross section: The shell fragments broken into small pieces and mounted with broken edges longitudinally facing upper most.

3) The shell membrane: From some fragments egg-membrane peeled and mounted the eggshell facing shell membrane the uppermost.

4) The inner most surface of shell adjacent to shell membrane: From some fragments the shell membrane was removed and such fragments of shell mounted with the inner surface of shell uppermost.

5) The inner surface of the egg membrane: The fragments of eggshell mounted with inner surface upper most.

All the mounted samples were labelled. Since the eggshell is a biological material which is non-conducting, it was coated with gold in Poleron Sputter Coater (England made) for 70 seconds to make it conducting. The thickness of coat observed as around $70 A^{\circ}$. The stub with coated material was scanned using Cambridge scanning electron microscope. The photographs were taken by automatic camera attached to the SEM unit on 100ASA Sakura Photofilm at suitable magnifications. The scanning was carried out at RSIC of Nagpur University Nagpur. The scanned material was observed critically and the structures are described in the following chapter.

\section{Observations:}

Hemiductylus flaviviridis is a common wall lizard found throughout the India. The female lays clutches of 4 to 7 eggs in the months of April to June, at dry and dark places. The eggs are spherical, rigid and calcareous in nature (Fig. 1). They do not swell during the period of incubation. The eggshells were scanned using SEM and to explain the morphological structure of eggshell, the terminology used is based on avian eggshell.

\section{The Radial Section :}

The radial fracture of eggshell has two distinct outer and inner layers. The outer layer is compact crystalline layer and the inner one is a fibrous shell membrane.

In $H$. flaviviridis also the outer calcareous crystalline layer does not show the differentiation of zones but it is not poorly developed (Fig. 2). It is thick and measures about $76 \mathrm{\mu m}$. The shell membrane is very thin and measures about $8 \mu \mathrm{m}$ in thickness.

In $H$. flaviviridis the calcareous crystalline layer of the eggshell is well developed and thick. It is composed of columnar shell units. These columns are slightly wide at outer surface and forming the blocks of crystalline material. On the outer most surface, no cuticle like organic material is found. The shell membrane is very thin (Figs. 2, 3,4). $\mathrm{m}$ size and shapes intermingling with fibrous structure (Fig. 5). The Outer Surface Of Crystalline Layer: The outer surface of eggshell of $H$. flaviviridis contains crystalline shell units of rando In $H$. flaviviridis the outer surface is different than that of $L$. punctata granosa and $C$. versicolor. It shows crystalline shell units of random size and shape (Fig. 5). The magnified view of the outer surface shows the presence of fibres on outer surface, along with the crystals (Figs. 6-7). Such structure is not present in earlier examined eggshells. $\mathrm{m}$ size and shapes intermingling with fibrous structure (Fig. 5).

The Inner Surface Of Crystalline Layer: The inner surface of eggshell of $H$. flaviviridis shows lower ends of shell units. The shell units are cones shaped. These cones protrude from mammillary caps. Between the columns very tiny fibres are seen (Figs. 8,9). After the removal of membrane the inner surface shows needle like crystals (Figs. 10,11). The fibres are absent.

\section{The Shell Membrane:}

The shell membrane of $H$. flaviviridis has entirely different structure. It is non-fibrous and porous. The membrane thickness is very less as compared to that of crystalline layer as well as the shell membranes of $L$. punctata granosa, C. versicolor and $C$. palustris. It is a sheet of organic matter pierced with pores of various sizes (Figs. 12, 13 and 14). 


\section{Discussion:}

The eggshells of Calotes versicolor and Hemiductylus flaviviridis have entirely different structural pattern. The presence of flexible or parchment-like porous eggshell in C. versicolor may facilitate the absorption of water from the soil to maintain the hydric condition during embryogenesis as reported in the turtle, Chelydra serpentina (Morris et al., 1983). On the contrary the eggshell of $H$. flaviviridis is hard, nonporous, calcareous and brittle, which may help in protecting the egg from desiccation, since the eggs are usually laid during hot summer (April-June) at dry and dark places inside the houses.

The eggs of oviparous lepidosauria usually have a flexible, parchment-like shell containing relatively small amount of calcium carbonate as observed in $C$. versicolor (Dendy, 1899 a, b; Bellairs, 1959; Dawbin, 1962; Sharell, 1966; Bellairs, 1970; Benoit, 1990). However, gekkonid lizards produce eggs with relatively hard calcareous shell which is common in the lizards of the family Dibamidae (Boulenger, 1912; Bustard, 1968 a). The variation in the structure of eggshells with change in parity mode was reported in gekkonid lizards by Girling et al.(1998). In Hemiductylus turcicus they have described the oviparous mode of reproduction and a hard calcareous eggshells, whereas Saltuarius wyberba, though exhibit oviparity produces a soft, parchment-like eggshell. The difference in eggshell structure with in the gekkonid lizards may be for maintaining the favorable water balance required for embryogenesis.

In lepidosauria, the eggshell of Urosaurus ornatus did not have a cuticle, whereas it was most apparent on the eggshells of Sceloporus undulatus hyancinthinus, S. clarkii and $S$. scalaris. Except for a thin 'cuticle' on the eggshell of Sceloporine species, a cuticle has not been reported on the eggshell of any squamate reptile (Mathies and Andrews, 2000). The cuticle as such is not present on the crystalline layer of eggshell of either $C$. versicolor or on the eggshell of $H$. flaviviridis.

Outer surface of eggshell of $H$. flaviviridis is formed by the crystalline blocks of uneven size and shape which are slightly widen at outer surface. These blocks are formed by the columner shell units. The presence of fibres intermingling with crystalline shell units on outer surface, between which crystals are seen is a unique feature, occurred only in the $H$. flaviviridis. Eggshells of other lepidosaurian, birds, crocodilians, and turtles also exhibit an intimate association between fibres of the shell membrane and calcareous layer, but only at the inner most tips of columns (Board, 1982; Ewert, 1985; Furguson, 1985; Packard and Hirsch, 1986). This arrangement is due to the sequential formation of the shell membrane and calcareous layer (Fujii and Tamura, 1970; Fujii 1974; Packard et al., 1984 b). Once the membrane has been formed, individual columns arise at specific nucleation sites and grow away from the membrane at a short distance and into the membrane ( Packard, 1980; Board, 1982; Packard et al., $1984 \mathrm{a}, \mathrm{b})$. The major direction of growth is away from the membrane, because the membrane itself seemingly limits the availability of calcium carbonate to forming columns.

The intimate and extensive association between the shell membrane and calcareous columns till the outermost surface propose that the calcareous layer and shell membrane form simultaneously, rather than sequentially, in $H$. flaviviridis and as a the columns are deeply rooted in the membrane. After the removal of very thin and closely associated shell membrane, the calcareous structure shows needle like crystal of calcium carbonate on the inner side.

\section{Shell Membrane:}

The $H$. flaviviridis lays the calcareous, brittle tiny eggshell, like that of gekkonid lizard. The shell membrane is nonfibriller and very thin and entirely different from that of the parchment-like eggshells ( Jacobi, 1936; Packard et al., 1977). An eggshell of $H$. flaviviridis shows intimate association between the crystalline and nonfibriller shell membrane. The tips of shell unit of columns protrude out through this thin layer of shell membrane. In contrast, a clear demarcation exists between fibrous and crystalline layers of eggshells of other reptiles and birds (Board, 1982; Ewert, 1985; Packard and 
Hirsch, 1986). Such evolutionary reduction in the shell membrane of lepidosaurians usually persists in varying degrees (Jacobi, 1936; Bellairs, 1959; Benoit, 1990). The phrynosonatia lizards show further reduction in the eggshell thickness to facilitate the exchange of respiratory gases (Blackburn, 1993, 1995). The shell membrane of $H$. flaviviridis is non-fibrous sheet of organic matter pierced with pores of uneven and various shapes and sizes. Thus it is structurally different from the most of the lepidosaurian.

\section{Summary:}

Hemiductylus flaviviridis, a wall lizard lays spherical, rigid and calcareous eggs. Eggshells of these four species have two distinct layers outer crystalline layer and inner fibrous shell membrane.

Outer Crystalline Layer: In $H$. flaviviridis the eggshell shows the outer calcareous, crystalline layer. The calcareous crystalline layer is thick. The crystalline layer is well developed and forms a column. These columns are widen slightly at outer surface and form a block of crystalline material. Outer most surface shows crystalline blocks of different size and shape. The outer structure shows the presence of fibres on outer surface, in between these fibres crystals are seen.

Shell Membrane: In $H$. flaviviridis the shell membrane is non-fibrilar porous and appear like that of sheet of organic material.

The fine structures of eggshells of four representative of different groups shows morphological variability. This structural variability may be due to their incubating environment. The details of structural modification in the turtle, lizards and crocodile certainly enhance the understanding of role they play during the development of embryo.

\section{References:}

Bellairs, A.d'A. (1959). Reproduction in lizard and snakes. New Biology, 30 : 73-90.

Bellairs, A.d'A. (1970). The life of reptiles. Vol. II Universe Books, New York.

Benoit, H. (1990). Comparative study of eggshell membrane among the oviparous and viviparous ancestor of the lizard, Lacerta vivipara. Can. J. Zool., 68(5) : 10151019.
Blackburn, D.G. (1993). Charioallantoic placentation in squamate reptiles : structure, function, development and evolution. Journal of Experimental Zoology. $266: 414-430$.

Blackburn, D.G. (1995). Saltationist and punctuated equilibrium models for the evolution of viviparity and placentation. Journal of Theoretical Biology, 174 : 199216.

Board, R.G. (1982). Properties of avian egg shells and their adaptive value. Biol. Rev. Combridge Philos.Soc., 57:1-28.

Boulenger, G.A. (1912). A vertebrate fauna of the Malay Peninsula from the Isthmus of Kra to Singapore including the Adjacent Islands, Reptilia and Batrachia. Taylor and Francis, London.

Bustard, H.R. (1968). The eggshell of gekkonid lizards : A taxonomic adjunct. Capeia, : 162-164.

Dawbin, W.H. (1962). The tuatara in its natural habitat. Endeavour, 21:16-24.

Dendy, A. (1899 b). Outline of the development of the tuatara, Sphenodon (Hatteria) punctatus. Quarterl Journal of Microscopical Science, 42(NS) : 1-87.

Ewert, M. A. (1985). Embryology of turtles. In C. Gans, F. Billett, and P.F. A. Maderson (eds) : Biology of the Reptilia Vol. 14, Development A. New York : John Wiley and Sons, PP. 75-267.

Ferguson, M.W.J. (1985). Reproductive biology and embryology of the crocodilians. In c. Gans, F. Billett and P.F.A. Maderson (Eds.) : Biology of the Reptilia, vol. 14, Development A. New York : John Wiley and Sons, PP. 229-491.

Fujii, S. and T. Tamura. (1970). Scanning electron microscopy of shell formation in hen's eggs. J. Fac. fish. Anim. Husb. Hiroshima Univ., $9: 65-80$.

Fujii, S. (1974). Further morphological studies on the formation and structure of hen's eggshell by scanning electron microscopy. J. Fac. Fish. Anim. Husb. Hiroshima Univ., $13: 29-56$.

Girling, J. E; A. Cree; JR. L. J. Guillette (1998). Oviducal structure in four species of gekkonid lizard differing in parity mode and eggshell structure. Journal Reprod. Fertility and Development, 10(2) : 139-154.

Jacobi, L. (1936). Ovoviviparie bei einheimischen Eidechsen. Verleichende Untersuchungen and den Eiern und am Ovidukt von Lacerta agilis, Lacerta vivipara und Anguis fragilis. Zeitchritt für Wissenschaft Liche Zoologie., 148 : 401464. 
Mathies,T. and R. M. Andrews (2000). Does reduction of the eggshell occur concurrently with or subsequent to the evolution of viviparity in phrynosomatid lizards? Biological Journal of the Linncan Society of London.

Morris, K. A.; G. C. Packard; T. J. Boardman; G. L. Paukstis and M. J. Packard (1983). Effect of Hydric Environment on Growth of embryonic snapping turtles Chelydra serpentina. Herpetologica, 39(3):272-285.

Packard, M. J. (1980). Ultrastructural morphology of the shell and shell membrane of eggs of common snapping turtle, Chelydra serpentina. Journal of Marphology, 165 : 187-204.

Packard, M. J. and K. F. Hirsch (1986). Scanning electron microscopy of eggshells of contemporary reptiles. Scanning Electron Miscrosc., IV : 1581-1590.

Packard, M. J. and G. C. Packard (1984). Comparative aspects of calcium metabolism in embryonic reptiles and birds. In R.S. Seymour (ed.) : Respiration and Metabolism of Embryonic Vertebrates. The Hague : Dr. W. Junk.

Packard, M. J.; L. K. Burns; K. F. Hirsch and G. C. Packard (1982 a). Structure of

\section{EGG OF LEPIDOSAURIA}

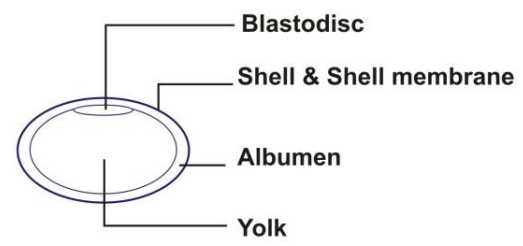

Fig. No.1:

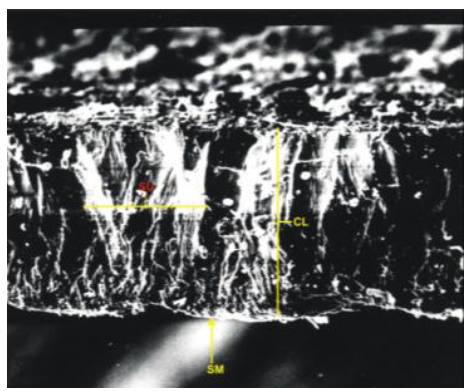

Fig. No.3 and 4:

Radial view of egg shell showing crystalline layer and thin indistinct shell membrane

shells from eggs of Zebra-tailed lizards, Iquanidae : Callisaurus draconoides. Zool. J. Linn Soc., 75: 297-316.

Packard, M. J; K. F. Hirsch and V. B. Meyerrochow (1982 b). Structure of the shell from eggs of the tuatara, Sphenodon punctatus. J. Morphol., 174 : 197-205.

Packard, M. J.; K. F. Hirsch and J. B. Iverson (1984 a). Structure of shell from eggs of kinosternid Turtles. Journal of Morphology, 181: 9-20.

Packard, M. J.; J. B. Iverson and G. C. Packard (1984 b). Morphology of shell formation in eggs of the turtle Kinosternon flavescens. J. Morphol., 181 : 21-28.

Packard, G. C.; C. R. Tracy and J. J. Roth (1977). The Physiological ecology of reptilian eggs and embryos and the evolution of viviparity within the class reptilia., Biol. Rev. 52 : 71-105.

Sahoo, G; B. K. Mohopatra; R. K. Sahoo; H. P. Mohanty (1996). Ultrastucture and characteristics of eggshells of the olive ridley turtle, Lepidochelys olivanceae from Gahirmatha, India. Acta-Anatomica, 156(4) : 261-267.

Sharell, R. (1966). The Tuatara, Lizards and Frogs of New Zealand. London : Collins.

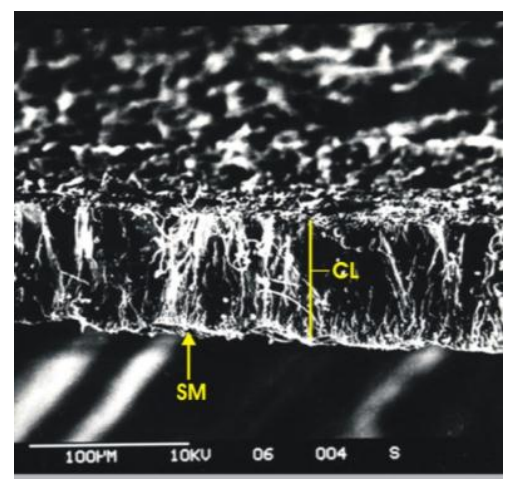

Fig. No.2 : Radial view showing thick crystalline layer and a verv thin shell membrane

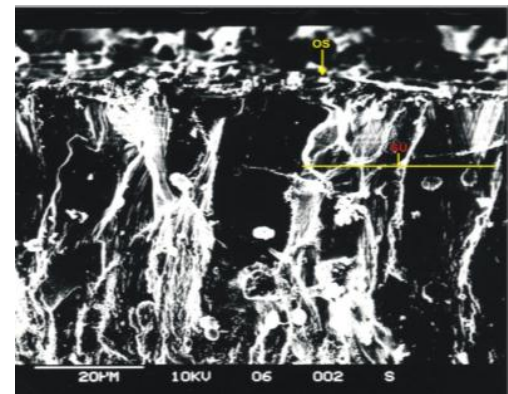




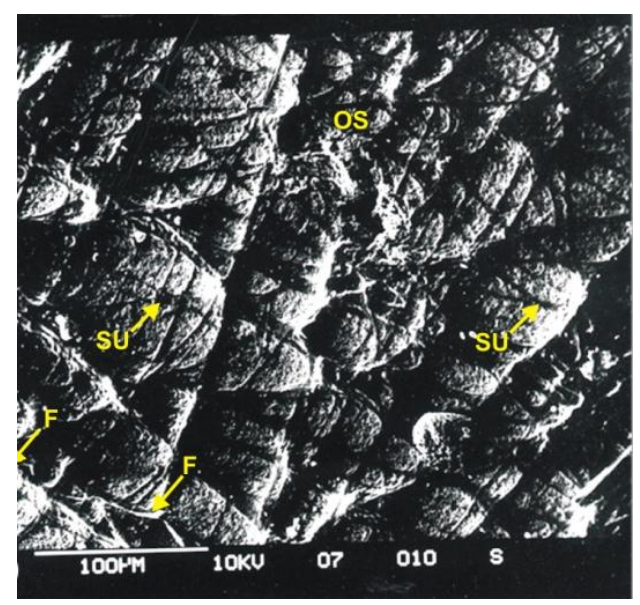

Fig. No.5 : Outer surface of egg shell showing blocks of shell unit.
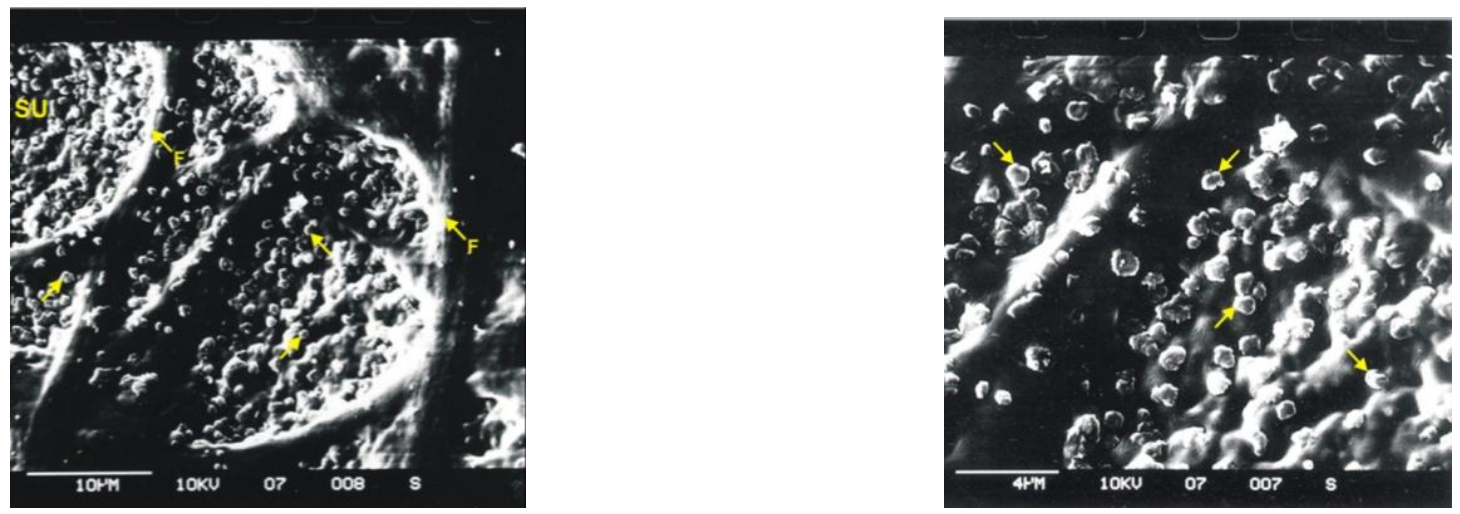

Fig. No.6 and 7: Magnified view of crystalline blocks of shell units showing crystalline deposites.
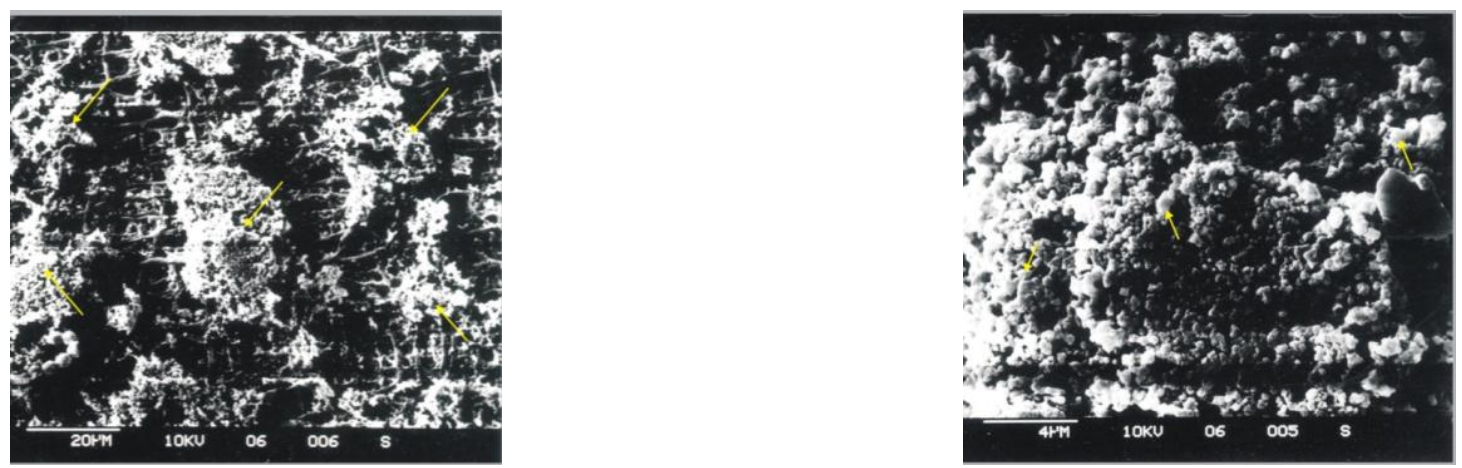

Fig. No.8 and 9: A view of lower end of shell units showing cone shaped protuberance associated with fibres. 

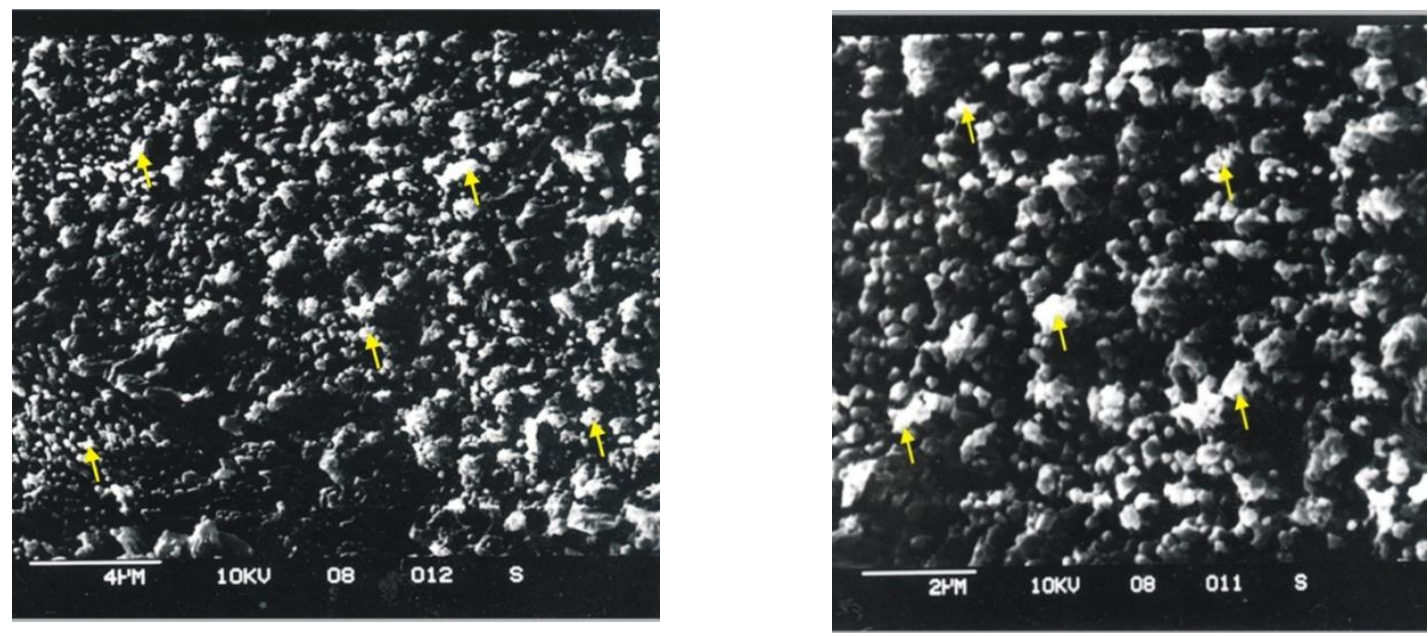

Fig. No.10 and 11: Innermost surface of egg shell after removal of egg membrane shows needle like crystals which forms shell units.

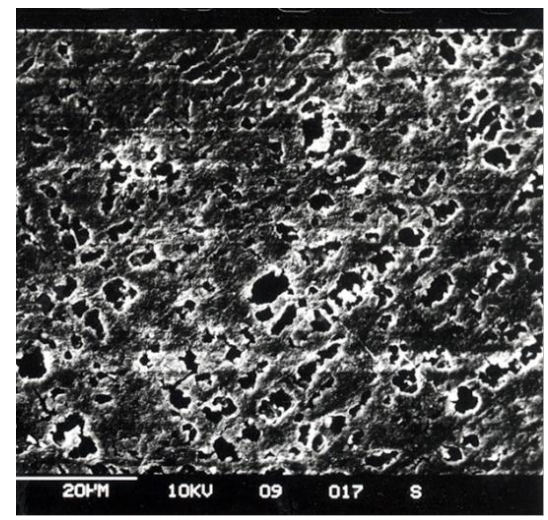

Fig. No.12: Shell membrane facing crystalline layer showing non -fibrillar structure
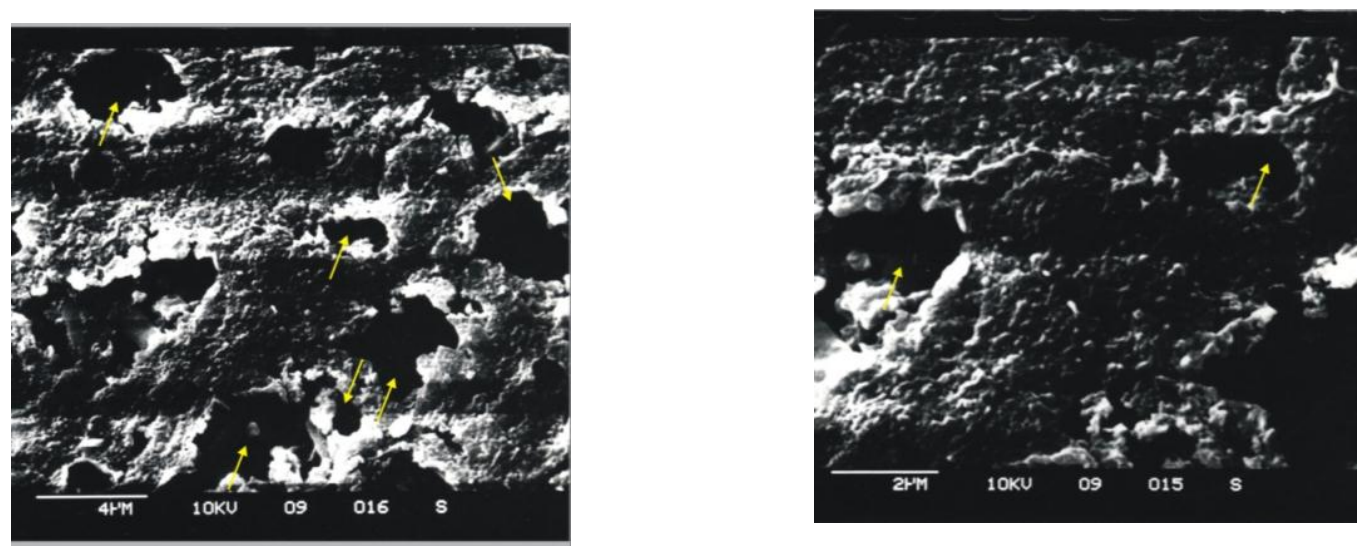

Fig. No.13 and 14: Magnified view of shell membrane showing non-fibrous structure with pores 